Derecho y Realidad

Vol.13 -Núm. 26 • Julio-Diciembre de 2015

Págs.171-184• ISSN:1692-3936

\title{
La defensa de los derechos sexuales y reproductivos de las mujeres indígenas de Colombia en la ONU*
}

\author{
The defense of sexual and reproductive rights of \\ indigenous women of Colombia in UNO
}

Anna Schmit**

\section{Resumen}

Colombia ratifica en el 1991 una nueva Constitución que reconoce, por una parte, el carácter multicultural de la nación, y por otro, la igualdad de las mujeres y de los hombres. Así, tiende a fijar las categorías sociales, lo que desfavorece el ejercicio de los derechos de las ciudadanas indígenas. Con el fin de remediar este hiato legislativo, una organización de defensa de derechos humanos de las mujeres indígenas propone en el 2012 reforzar una alianza entre las mujeres indígenas del país, alrededor de la elaboración de su primer informe sombra (shadow report) ante el Comité para la Eliminación de las Discriminaciones hacia las Mujeres (CoEDEF). En esa acción, las mujeres

Fecha de aceptación: 2 de agosto de 2015

Concepto de recepción: 22 de agosto de 2015

Fecha de aprobación: 15 de septiembre de 2015

* Este articulo esta sacada de la ponencia de la autora en el Congreso de derechos humanos de la UPTC que se dio en Tunja los dias 21, 22 y 23 de octubre del 2015.

** Doctorante de la Escuela de Altos Estudios de Ciencias Sociales de París (EHESS) en Antropología Social. Forma parte del equipo de investigación del LAIOS (Laboratorio de Antropología de las Instituciones y Organizaciones Sociales) y del IIAC (Instituto Interdisciplinario de Antropología de lo Contemporáneo). aschmita@gmail.com 
trataron de construir sus derechos sexuales y reproductivos como un sujeto de interés común que necesitaba un acompañamiento gubernamental. Este artículo propone vislumbrar los efectos de esta acción colectiva en la construcción de un posicionamiento político común a las mujeres indígenas y en la recepción del informe sombra por el Gobierno y las expertas del Comité.

\section{Palabras clave}

Acción colectiva, mujeres indígenas, derechos sexuales, Organización de las Naciones Unidas.

\section{Abstract}

In Colombia rules since 1991 a new Constitution that recognizes, on the one hand, the multicultural character of the nation and, on the other one, the equality of women and men. Thus, it tends to produce a boundary between social categories, which discourages the exercise of the rights of the Indigenous female citizens. To remedy this legislative gap, in 2012, a local association of indigenous women's human rights proposed to strengthen the alliance between the indigenous women of the country, around its first shadow report, which was presented to the Union Nation Comitee for the Elimination of Discrimination against Women (CoEDAW). During this collective action, indigenous women try to raise their sexual and reproductive rights as a subject of common interest that requires Government support. This article has two aimes. Firstly, it seeks to study the effects of this referal on the construction of common political position of Indigenous women. Secondly, it seeks to analyze the response to this same referal on the part of Colombian State and the CoEDAW.

\section{Keywords}

Collective action, indigenous women, sexual rights, Colombia, Union Nations Organization. 


\section{Sumario}

1. De la victimización indígena a la subjetivación feminista

2. Movilización indígena en torno a la CEDAW

3. Construcción de la voz de las mujeres indígenas en la ONU

4. Conclusiones y referencias

\section{Método}

Jurídico Social.

\section{Metodología}

En este texto se resalta la importancia de la mujer y sus derechos sexuales y reproductivos, pero como reponsabilidad del estado colombiano de la defensa de estos ante la ONU, por las violaciones a las comunidades indígenas. 


\section{Introducción}

Este artículo presenta el proceso por el cual las mujeres indígenas de Colombia construyeron sus derechos sexuales y reproductivos como uno de los ejes de sus demandas ante el Comité de Naciones Unidas para la eliminación de la discriminación hacia las mujeres (CoEDAW), que examinó el caso colombiano en octubre del 2013. También expone como las expertas de este Comité elevaron esas demandas y si el Estado colombiano las escuchó o no.

En este examen periódico, el Comité se apoya en el informe oficial, hecho por el Estado, para cuestionarlo, estimular y dirigir sus esfuerzos a la aplicación de la Convención de Naciones Unidas para la Eliminación de las Discriminaciones hacia las Mujeres (CEDAW). A raíz del diálogo que se establece entre ambos, el Comité formula recomendaciones que son susceptibles de influir la orientación de las políticas públicas llevadas por los gobiernos de turno. Las organizaciones de la sociedad civil están invitadas a presentar un informe sombra, que responde al informe oficial para dar cuenta a las expertas del Comité de su situación real, además pueden observar el examen sin intervenir.

En Colombia, la Constitución reconoce la igualdad entre hombres y mujeres. También admite a los pueblos indígenas el ejercicio de una gobernanza autónoma y el goce de sus derechos colectivos. Los movimientos de mujeres, como las organizaciones indígenas, están invitados a participar en el juego político mediante las mesas de concertación (MPC). A pesar de esos avances normativos, la interpretación de la Constitución y la formación de las MPC tienden a fijar las categorías sociales según si se trata de los derechos de las mujeres o de los derechos de los indígenas. La rigidez de esas categorizaciones tiende a excluir a las ciudadanas indígenas de los órganos democráticos, lo que obstaculiza su protección. Frecuentemente, las mujeres indígenas están sometidas a distintos tipos de violencia (social, armada, familiar), sus derechos sexuales y reproductivos no son respetados y su acceso a la justicia es demasiado precario para pretender una protección eficiente.

Para remediar esa situación, una asociación local de defensa de los derechos colectivos indígenas, la Fuerza de Mujeres Wayuu, ubicada en La Guajira, inició una acción colectiva, inédita y común a las mujeres indígenas del país. Se trata de la formulación de un informe sombra ante el Comité de la CEDAW. Esa acción es inédita, porque por primera vez en Colombia, las mujeres indígenas deciden acudir a un dispositivo internacional garante de los derechos de las mujeres.

La elaboración de un informe sombra por las mujeres indígenas debe entenderse como un proceso a través del cual las mujeres tratan de constituirse en sujeto 
político, apto para participar en el juego democrático de su país. Ellas posicionan sus derechos sexuales y reproductivos en el centro de los debates, y denuncian las prácticas de mutilación genital, que persisten especialmente en algunas comunidades emberas. La cuestión de los derechos sexuales y reproductivos y la del dominio de su sexualidad por y para ellas, forman una cuestión política de autonomía del sujeto "mujer indígena", que tratan de consolidar en esta ocasión (Del Re, 2007).

Este artículo tiene dos objetivos. El primero es entender el proceso que impulsa a las mujeres indígenas a acudir a los derechos de las mujeres y que les incita a construir los derechos sexuales y reproductivos en un sujeto de intereses comunes que necesita una intervención pública. El segundo objetivo es entender como la delegación colombiana que representa al Estado colombiano hace caso de sus reivindicaciones en la sesión de la ONU. Nos apoyamos en datos etnográficos identificados durante un trabajo de campo hecho con la Fuerza de Mujeres Wayuu entre septiembre 2012 y noviembre $2013^{1}$.

\section{De la victimización indígena a la subjetivación feminista}

\section{Formación de un colectivo de víctimas FMW}

El proyecto de elaboración del informe sombra por y para las mujeres indígenas, fue introducido en el movimiento indígena de Colombia, por la Fuerza de Mujeres Wayuu. Es una organización de defensa de los derechos humanos de los hombres y de las mujeres indígenas, definida por sus miembros como un movimiento asociativo. Ese colectivo nace en el 2006, para formar un movimiento de víctimas, decidido a denunciar los crímenes paramilitares y a exigir al Estado una reparación colectiva, basada en la visión wayuu de lo que debe ser el enfoque diferencial.

Los miembros del colectivo son, en su mayoría pero no exclusivamente, mujeres. Al principio construyeron un discurso victimario que se apoyaba en la pertenencia indígena. Poco a poco, la sobrerrepresentación femenina acabó por atraer la atención de organizaciones feministas de alcance internacional, por ejemplo el Fundo Global por las Mujeres. En el 2009, una organización noruega feminista -FOKUS- invita a Fuerza a integrar un programa que tiene el objetivo de mejorar las capacidades organizativas de diversas asociaciones de mujeres en un contexto de conflicto armado, y pretende capacitar a los colectivos de mujeres sobre sus derechos y ponerlos en red. Para integrar este proyecto, la FMW tuvo que constituirse como una organización oficial, definida por estatutos registrados en la cámara de comercio.

\footnotetext{
Agradecemos al IFEA (Institut Français d'Etudes Andines) et el SOGIP (Observatoire des échelles de gouvernances, les Nation Unies et les peuples autochtones) que apoyaron el trabajo de campo.
} 


\section{El momento de la subjetivación y la inclusión del derecho de las mujeres}

La trasformación del movimiento de víctimas en una ONG y la incorporación de una perspectiva de género, obliga a las militantes a iniciar una reflexión en torno de los medios que deben adoptar para garantizar los derechos de las mujeres indígenas. Las militantes empiezan a cuestionar su pertenencia indígena a través de un lente de género, y a nombrar las violencias que sufren bajo los términos genéricos "violencia de género" o "violencia contra las mujeres". De manera progresiva, un discurso político se formaliza alrededor de una identidad social intersecional, hecha por el cruce de categorías subalternas "mujer" e "indígena" (Crenshaw \& Bonis, 2005, p. 51-82; Purtschert \& Meyer, p. 127-146).

Las alianzas establecidas con FOKUS sugieren a las militantes un modelo de desciframiento de una situación personal a la luz de una lectura de género. Por lo tanto, estimulan un proceso de subjetivación a partir del cual las militantes de la FMW promueven los derechos de las mujeres. La subjetivación define un proceso de adquisición de elementos exteriores que un sujeto vuelve subjetivos. Así, participa en la construcción de una narración colectiva que da sentido a las historias individuales. El sujeto esta entendido aquí, según las palabras de Eric Tassin, como "un grupo, involucrado en un proceso de emancipación" (Tassin, 2014, p. 157-173). Las militantes de las FMW empiezan a analizar los efectos del conflicto armado sobre sus vidas, no solamente a la luz del racismo estructural que persiste en Colombia en contra de los pueblos indígenas, sino, también, incorporando una perspectiva de género, en la cual articulan las violencias cometidas por actores armados y las que persisten en las comunidades.

En el 2010, la fundadora de la FMW sigue la formación sobre la CEDAW, que propone en Toronto el Instituto de Educación para los Derechos de las Mujeres (WHRI). Del encuentro entre la militante y el equipo de la formadora, surge la idea de organizar la llegada del Instituto a Colombia, para propiciar una formación sobre los derechos de las mujeres, especialmente dirigida a las mujeres indígenas. Aprovechan la agenda de la ONU, que proporciona el examen de Colombia por el Comité EDAW en octubre del 2013. Finalmente, el Instituto llega en septiembre del 2012.

\section{Movilización indígena en torno a la CEDAW}

\section{Cronología de la acción}

El informe sombra se construye en distintos momentos, en diversas reuniones y eventos, durante los cuales la estructura y el contenido del informe son debatidos y se van definiendo. Estas reuniones fueron calificadas como "eventos" por las mujeres, por eso las Ilamaremos así en este artículo. Hubo seis eventos claves: 
1) EI primero fue durante la primera semana CEDAW para las mujeres indígenas, organizado por la FMW con el Instituto y FOKUS, en el Cabo de la Vela.

2) El segundo evento se dio con ocasión de un conversatorio para las mujeres indígenas sobre la aplicación del Auto 092, organizado por la ONIC en la Costa Caribe. Este reunió unas 40 mujeres en La Mina, territorio Kankuamo.

3) El tercer evento había sido decidido durante la primera semana. Era "El encuentro de las mujeres indígenas para la elaboración del IS", organizado por el CRIDEC, y que tuvo lugar en Río Sucio, Caldas, durante el 26 y 27 de noviembre. Reunió unas veinte mujeres.

4) La Cumbre Ideológica de los Pueblos reunió todos los pueblos de Colombia entre el 10 y el 13 de febrero en territorio Zenú (Sucre). La ONIC, a través de su Consejería Mujer, Familia y Generación, puso el informe sombra en el orden del día e invitó a la OPIAC y al AICO a seguir. Pero, en ese momento, el proyecto no ganó la adhesión de todas y se quedó en suspenso hasta:

5) 2013, en abril, con ocasión de una mesa de concertación, en la que hubo un almuerzo informal entre la ONIC, Ia OPIAC, el AICO y la FMW, para discutir acerca de los asuntos logísticos.

6) Esta serie de reuniones se finaliza en mayo del 2013, con "el encuentro de las mujeres indígenas para la elaboración del IS", que se hizo en territorio kankuamo, del 23 a 25 de mayo de 2013. Este último evento reunió unas 20 participantes.

\section{Justicia y sexualidad}

Durante todos los eventos, las mujeres indígenas trataron de acordar los temas que debía contener el informe sombra. Todas estaban de acuerdo con denunciar su débil participación política, pero dos temas aparecían conflictivos. El primero se refería a las violencias cometidas contra ellas en las comunidades, específicamente la violencia sexual, y el segundo, la precariedad de su acceso a la justicia, que no les permitía denunciar estas violencias. Esos temas cuestionan no solamente el sentido de las políticas nacionales multiculturales, sino también la voluntad indígena de reproducir o no ciertas tradiciones a las cuales las mujeres oponían sus derechos humanos. Por ejemplo, las mutilaciones genitales que se practican en algunas comunidades emberas eran debatidas y las mujeres coincidían sobre la necesidad de denunciarlas y de acabarlas. Una militante embera del CRIDEC explica así durante el tercer evento:

En el departamento de Caldas, nos hemos metido en el tema del acto de la ablación para las niñas y nosotros hemos dicho que eso es un acto de barbarie más allá de considerar que es algo cultural. Aquí tenemos información, hace 15 días se sangró una niña mientras le estaban haciendo el trabajo de ablación y se murió. Nosotros hemos dicho que este hecho era gravísimo. 
La intervención gubernamental para garantizar el acceso de las mujeres indígenas a la justicia, también formaba un tema de disenso. Las mujeres indígenas consideraban que si no estaban protegidas dentro de sus comunidades en contra de las distintas formas de violencia que podían vivir, entonces estaban en una situación de aguda vulnerabilidad frente a actores exteriores, armados. Exigían que la justicia indígena, en coordinación con la justicia ordinaria, tomara en cuenta estas violencias. No pedían al Estado sustituir a las autoridades indígenas, sino acompañarlas para considerar estos problemas, y propiciar espacios de diálogos en los cuales estos temas podían ser discutidos y debatidos con las autoridades indígenas. Una participante dice así:

Nuestro discurso debe ser más coherente. Tenemos que delimitar las intervenciones del Estado. Como indígenas podemos tomar medidas, lo que queremos es que el Estado garantice que estas medidas se tomen. Por ejemplo, si decimos: "este año tantas niñas fueron violados por sus abuelos, bueno, ¿qué hacemos con los abuelos?" Cómo el Estado puede ayudar a la comunidad para arreglar este tipo de problemas y garantizar que esas cosas no se producen?

Sin embargo, la Cumbre Ideológica de los Pueblos provocó una ruptura en los esfuerzos para constituir la violencia intracomunitaria como un sujeto de interés común que necesitaba una intervención gubernamental. Era difícil motivar la adhesión de las organizaciones nacionales, sobre todo la ONIC y el AICO, alrededor de la denuncia franca y crítica de lo que podía suceder en las comunidades. Esas organizaciones temían ir en contra del movimiento indígena, si estos hechos se denunciaban en un espacio internacional. En una primera versión del informe sombra, todos los rasgos de este violencia intracomunitaria habían sido borrados. La fundadora de la FMW le hizo una corrección, integrando una denuncia más amplia de las violencias cometidas en contra de las mujeres indígenas, identificando las prácticas que:

(...) nos discriminan cuando a partir de posturas "culturalistas" justifican "usos y costumbres" que terminan lesionando nuestras vidas y dignidad, como es el caso de la practicas nocivas como la mutilación genital, la pelona (rito en el que se arranca el cabello a tirones a las niñas que entran a la pubertad), la violencia, los mal tratos, los matrimonios con niñas, el incesto, limitaciones y en algunos casos prohibiciones para la participación política, entre otras prácticas. (Informe sombra de las mujeres indígenas, 2013, p.17)

En septiembre del 2013, la FMW coordinó la redacción del informe sombra hecho por las mujeres indígenas: Derechos humanos, discriminación y violencia: una mirada a la situación que enfrentamos las mujeres indígenas en Colombia. 
La ONIC estableció una alianza con el movimiento de mujeres para incluir la situación de las mujeres indígenas en el informe general Una mirada a los derechos de las mujeres en Colombia, hecho por una coalición de organizaciones colombianas.

Según la coordinadora del Programa Presidencial por los Derechos Humanos, presente en la sesión en Ginebra, las instituciones no tuvieron conocimiento del IS hecho por Fuerza, lo que reduce sustancialmente sus efectos.

\section{Construcción de la voz de las mujeres indígenas en la ONU}

Las observaciones en la sesión de la ONU se dividieron en tres tiempos: 1. Una reunión informal entre las mujeres que componían la delegación indígena: dos militantes de la FMW y dos de la ONIC, una mujer nasa y una joven embera de Risaralda. 2. El "almuerzo informal" o la presesión, que consiste en un encuentro entre las organizaciones de la sociedad y las expertas del Comité, para que las primeras expongan a las segundas lo que les parece más importante y que respondan a las dudas de las expertas. 3. La sesión.

\section{Politizar la sexualidad en las Naciones unidas}

Antes de que empezara la presesión, la delegación indígena se reunió con el fin de preparar su intervención ante las expertas del Comité. Se trataba de decidir los temas que merecían la atención de las expertas, exigir respuestas claras por parte del Estado, y recomendaciones del Comité. Abordaron la cuestión de su sexualidad. Consideraban que el control sexual al cual estaban sometidas era el principal vector por el cual pasaban las discriminaciones que sufrían. Les importaba obtener el apoyo del Comité para rectificar esta situación, por ejemplo a través de programas gubernamentales de educación sexual en las comunidades indígenas. Estimaban que la domesticación de sus cuerpos, que consagraba la ausencia de placer como una garantía del mantenimiento de las tradiciones, restringía su desarrollo personal. Esta prohibición se encontraba exacerbada en unas comunidades indígenas, en las cuales se practicaban mutilaciones genitales y que las mujeres presentes estaban dispuestas a denunciar.

Estaban de acuerdo sobre la importancia de establecer las mutilaciones genitales como un sujeto de atención prioritaria. Esto requería coordinar sus intervenciones durante la presesión y elaborar un vocabulario apropiado y claro para las expertas. Estaban conscientes de que ese tipo de mutilación era un tema muy delicado, porque podía ser mal interpretado y podía volverse en un potente obstáculo a la autonomía de los pueblos (Mathieu \& Echard, 2014). Con el fin de evitar eso, era imprescindible, para las mujeres, adoptar una perspectiva pedagógica en la cual explicaran al Comité que los esquemas culturales debían ser debatidos dentro de 
los pueblos y que no podían ser impuestos por agentes exteriores. Tenían que insistir sobre las razones que empujaban a las parteras a practicar la ablación. Frecuentemente se trataba de mujeres ancianas que no tenían consciencia de cometer una grave violación a los derechos humanos de las mujeres. Al contrario, estaban convencida de los cuidados que daban a la recién nacida. El clítoris era asimilado a un órgano masculino que entraba en competencia con el pene de los hombres $y$, por eso, podía ser responsable de desequilibrio mental para las mujeres. Según esta creencia, quitar el clítoris cancelaba la competencia y protegía a las mujeres de la locura.

Según las indígenas, su intervención ante las expertas de la ONU debía conducir a que el Comité orientara al Estado para promover debates que cuestionaran los méritos de esas prácticas en las comunidades, sin agudizar la estigmatización de las parteras en la sociedad nacional.

\section{De los sexos mutilados a la palabra astillada}

Después de esa reunión, la delegación indígena se unió al conjunto de organizaciones civiles colombianas para la presesión, la cual tiene un tiempo limitado de una hora. Ese momento sirve para que las actoras de la sociedad civil trasmitan los temas que quieren ver plasmados en el diálogo y que esclarezcan las dudas de las expertas sobre puntos precisos. Varias organizaciones de la sociedad civil colombiana estaban presentes. La representante de la Comisión Colombiana de Juristas había sido designada para mediar los debates. Estaba encargaba de administrar el tiempo de palabra de cada uno.

Los debates se centraron rápidamente sobre la sexualidad de las mujeres y varias veces las mujeres indígenas indicaban que tenían algo que decir. Pero, solo tuvieron la palabra al final del encuentro y exclusivamente para responder a una pregunta que les formuló una experta. La militante de la FMW aprovechó ese tiempo para introducir los problemas que enfrentaban las mujeres indígenas para acceder a los órganos de justicia. En el último minuto del encuentro, las indígenas alzaron su voz para plasmar las mutilaciones genitales en el debate y formular unas solicitudes muy claras al Comité:

Les queremos dar informaciones sobre las mutilaciones que se están produciendo en ciertas comunidades indígenas de Colombia. Consideramos que el Comité tiene la competencia para trabajar con nosotras para acabarlas. No se trata de considerar a las parteras como criminales. Es por su falta de conocimiento que practican la ablación del clítoris. Necesitamos de su apoyo y queremos que una recomendación esté dirigida a las instituciones nacionales con el fin de que las tomen en cuenta en los programas de políticas públicas. 
La gravedad de los hechos en el momento en el cual se terminaba la presesión, dio lugar a una reunión informal entre la experta relatora de la sesión colombiana y las mujeres indígenas. La relatora empezó por decirles que era preferible usar el término de "mutilación" al de "ablación". Enseguida, propuso tomar como instrumento de acción el artículo 5 de la Convención, relativo a la modificación de patrones socioculturales para lograr una igualdad entre hombres y mujeres, y de emitir una sanción en contra del Estado colombiano si no tomaba medidas. El Comité en sí no puede imponer sanción, pero puede sugerir a los otros órganos de Naciones Unidas que sometan las ayudas internacionales al respeto de una de sus recomendaciones. Las mujeres indígenas rechazaron esta opción que podía penalizar a su comunidad. Para ellas, esta opción podía acentuar la estigmatización de la medicina tradicional y de las parteras, lo que Ilevaría al rechazo de la sanción en las comunidades y, por lo tanto, las prácticas no bajarían. Reiteraron su solicitud de propiciar espacios de diálogos entre las comunidades y el Estado.

La descripción de la presesión muestra dos aspectos de la intervención de las indígenas. La primera es la dificultad que ellas tuvieron para inscribir sus reivindicaciones en el espacio de la presesión. Pudieron tomar la palabra cuando ya era tarde, lo que muestra la jerarquización implícita de los temas que necesitan una intervención del Comité. También muestra las discriminaciones estructurales que les toca y sus posiciones marginalizadas en el movimiento feminista colombiano.

El segundo aspecto ilustra la determinación de las mujeres indígenas para inscribir las mutilaciones en las prioridades del Comité, con el fin de convertirlas en una preocupación común y una prerrogativa de los programas públicos. Inscribieron así la integridad y la autonomía corporal de las mujeres en una "relación de poder", en la cual se esperaba que el Comité modificara los términos ${ }^{2}$. Haciendo eso, reafirmaban la misión del Estado de garantizar la integridad física y moral de las ciudadanas indígenas, que se encontraba en peligro por las prácticas de mutilación genital.

\section{Sexualidad y mujeres indígenas en el diálogo entre el Estado colombiano y el Comité}

En la sesión, la delegación colombiana estaba compuesta por diez mujeres, que trabajaban en instituciones y ministerio nacionales: ACPEM, Programa Presidencial para Ios Derechos Humanos, Ministerio de Relaciones Exteriores, Ministerio del Interior, Ministerio de la Protección Social, la Unidad Administrativa para la

$2 \quad$ En ce qui concerne les liens entre le Comité et le corps des femmes, voire le chapitre 7 " Le corps des femmes. Autonomie et intégrité corporelle dans la Convention ” in Roman (2014). 
Atención a las Víctimas, la Fiscalía, y la representante de la misión permanente de Colombia en Naciones Unidas.

La relatora del Comité demandaba al Estado colombiano rendir cuentas sobre las mutilaciones genitales que se practicaban en ciertas comunidades indígenas y que el informe no contemplaba. La directora del ACPEM subrayaba el trabajo que efectuaba el ICBF desde el 2007 e insistía en el hecho de que ni el Estado colombiano, ni la sociedad nacional, estaban enterrados de esas prácticas que calificaba de "místicas". Aprovechaba para recordar a las expertas el papel de las parteras en la reproducción de esa tradición. Según ella, las instituciones colombianas conocían los casos solo cuando la situación se revelaba muy peligrosa y requería transferir a la bebé a un centro de salud. Precisaba que las comunidades debían decidir sobre las eventuales sanciones, porque según los principios de Constitución, las autoridades indígenas debían regular sus normas. Así, subrayaba que el Estado no tenía la competencia para arreglar esos asuntos. Sin embargo, mencionó el trabajo de sensibilización que el Gobierno había empezado con las autoridades tradicionales y que Ilevó a que ellas reconocieran el carácter nocivo de dichas prácticas.

La respuesta de la funcionaria de la ACPEM no convenció a las expertas, que le pidieron más precisión sobre la estrategia de los gobiernos para erradicar las mutilaciones. Reprochaban la falta de datos cifrados, que consideraban de primera importancia para la aplicación y el seguimiento de políticas. La funcionaria siguió insistiendo sobre la responsabilidad de los indígenas y sobre las dificultades para el Gobierno de producir cifras:

Quiero reiterarles que sí es una preocupación del Gobierno colombiano. Sin embargo reitero, es muy difícil en este momento poder tener y brindar una información acertada de cual ha sido el registro. ¿Por qué? Porque estas comunidades están ubicadas en zonas de muy difícil acceso, no están cerca ni de un centro de salud ni de hospitales. Por lo tanto, los centros de salud no pueden tener conocimiento de esas prácticas, por lo cual el Ministerio de Salud en cabeza de las secretarias de salud departamentales y municipales no tienen un registro concreto más que los casos que presentan complicaciones y que tienen que ser trasladados a un hospital para precisamente evitar que tengan una situación mucho más lamentable. El trabajo ha sido más que todo de sensibilización porque esa práctica se hace a escondidas, no se hace a la luz pública, sino en el parto y los partos no son atendidos por los médicos sino por la comunidad misma con su medicina tradicional. Entonces, no podría darles de manera acertada mayores datos más que el interés de darle todo el seguimiento que estamos ya haciendo de los casos que ya conocemos. También, podría decirle que no es una práctica permitida. Hay un decreto en el cual las mismas autoridades indígenas están pidiendo sanciones severas a quienes cometen esa práctica. 
Así ocurrió lo que temían las mujeres indígenas, el discurso de la funcionaria pública insistía en la responsabilidad de las autoridades y de las parteras. Según ellas, este tipo de discurso conducía inexorablemente a agudizar la marginalización de las indígenas dentro de los espacios internacionales.

Al final de la sesión, las expertas destacaron la violencia intrafamiliar. La relatora cuestionaba los nexos entre la justicia y la violencia, insistiendo en la profusión de la violencia privada y la alta impunidad que tenía. Solicitaba información sobre cuáles eran las medidas de protección previstas para las mujeres indígenas, y cuáles eran los órganos de la justicia a los cuales podían acudir. La representante de la Fiscalía dio una respuesta breve y confusa, explicando que la competencia era de la justicia ordinaria pero que, de acuerdo con los principios de la Constitución, era preferible asignar esos casos a la justicia indígena, si se producían en sus territorios. Esa respuesta dejaba fuertes dudas sobre las posibilidades de recursos para la justicia de las mujeres indígenas.

Esos ejemplos muestran la naturaleza del diálogo que se establece entre las mujeres indígenas y los representantes del Estado colombiano bajo la mediación del Comité. Nos invitan a pensar "el Estado", como lo sugiere Sebastián Roux, sobre sus paradojas que lo balancean entre la obligación de protección y la real exclusión de sus ciudadanas, pero más bien como "lugar de producción de las identidades legítimas" (Roux, 2014, p. 4-10), en el cual, a través del informe sombra, las mujeres indígenas intentan entrar. La delegación colombiana rechazó de su competencia las demandas de un grupo de ciudadanas, canceló su voz excluyendo su demanda del marco de su competencia. Sin embargo, la intervención de las indígenas al lado de las expertas, y su presencia en esa sesión, dieron visibilidad a sus reivindicaciones e inscribieron su palabra en un espacio hegemónico (Spivak, 2009, p. 98), del cual se espera que favorezca su visibilidad ante el Estado.

\section{Conclusión}

Para concluir, el análisis del examen del caso colombiano ante el CoEDAW muestra los límites del espacio de las Naciones Unidas en el cual se tramite la reivindicación de la sociedad civil, que no está plenamente contemplada por el Estado. Sin embargo, la acción colectiva tiene el mérito de haber estimulado a las mujeres a formular sus problemas y, por lo tanto, a hacerlos existir, a difundirlos ante las instituciones públicas y a hacerse reconocer en un espacio público como sujeto político capaz de participar en la elaboración de las políticas públicas que les concierne. 


\section{Referencias}

Crenshaw, K. W. \& Bonis, O. (2005). Cartographies des marges: intersectionnalité, politique de l'identité et violences contre les femmes de couleur ", Cahiers du genre, 39(2), 51-82.

Del Re, A. (2007). Avortement et contraception. In Dictionnaire Critique du féminisme, (2e édit.). (pp. 1-6). Paris: PUF.

Mathieu, N. C. \& Echard, N. (2014). Les mutilations du sexe des femmes. In L'anatomie politique /I. Texte paru en 1982, sous la rubrique " Tribune libre " dans le Bulletin de I'AFA (Association françaises des anthropologues).

Purtschert, P. \& Meyer, K. (2009). Différences, pouvoir, capital. Réflexions critiques sur I'intersectionnalité. In: E. Dorlin (dir.), Sexe, race, classe. Pour une épistémologie de la domination, (pp. 127-146). Paris : PUF, ("Actuel Marx").

Roman, D. (2014). La Convention pour I'Elimination des Discriminations envers les femmes. Paris: Pedone. 370p.

Roux, S. et al. (2014). Penser I'État. Actes de la recherche en sciences sociales, 1(201-202), 4-10. DOI 10.3917/arss.201.0004

Spiivak, G. (2009). Les subalternes peuvent elles parler? Paris: Edition Amsterdam, $110 \mathrm{p}$.

Tassin, E. (2014). Subjectivation vs sujet politique: réflexion à partir d'Arendt et de Rancière. Tumultes, 2(43), 157-173. 